\title{
DEVELOPMENT OF BALLOON-BASED ORGAN RETRACTOR FOR LAPAROSCOPIC SURGERY
}

\author{
YOSHIHIRO KONDO*, DAISUKE TANIGUCHI, \\ KEITARO MATSUMOTO, TAKESHI NAGAYASU \\ and MURRAY JOHN LAWN \\ Nagasaki University Graduate School of Biomedical Sciences \\ 1-12-4 Sakamotomachi, \\ Nagasaki-shi, Nagasaki 852-8523, Japan \\ *y-kondo@nagasaki-u.ac.jp \\ IKUO YAMAMOTO \\ Department of Mechanical Science, Nagasaki University Graduate School, \\ 1-14 Bunkyomachi, Nagasaki-shi, Nagasaki 852-8521, Japan \\ iyamamoto@nagasaki-u.ac.jp \\ Received 13 January 2017 \\ Accepted 20 June 2017 \\ Published 12 October 2017
}

\begin{abstract}
Effective organ retraction is essential to ensure safe and efficient surgery, in the case of laparoscopic surgery, organ retraction is particularly difficult to achieve. Currently such as cherry dissectors are used for retraction, however the small size results in excessive stress on the organ being retracted. Therefore, we are developing a balloon-based retractor that provides similar functionality as the cherry dissector, particularly being able to pass through a small port, yet being expandable to provide a much greater contact area as well as improved traction, thus providing increased operating efficiency and improved safety in organ retraction. In this paper, the relative merits and demerits of a number of currently available retractors are compared. Parameters under consideration include shape, size, effective traction (coefficient of friction), type of actuation and ease of use. While parameters such as pressure distribution and effective traction have been objectively measured, other attributes unique to the individual mechanisms are discussed, and finally feedback is provided from surgeons regarding the respective retractor's ease of use and perceived efficacy.
\end{abstract}

Keywords: Balloon based organ retractor; frictional coefficient of retraction

\section{Background}

Surgical procedures in the abdominal, pelvic or thoracic cavities have traditionally involved open surgery. In this case, retraction of organs is relatively easy, organs may be retracted by hand or with the assistance of any convenient surgical device. ${ }^{1,2}$ However, in recent years, laparoscopic surgery has become the preferred choice of surgery where it is feasible as patient discomfort and recovery are in part related to the extent of the surgical incision/s. However, in the case of laparoscopic surgery, organ retraction is inherently difficult due to all surgical devices being required to pass through typically $5 \mathrm{~mm}$ or $10 \mathrm{~mm}$ trocars and the resulting lack of dexterity. ${ }^{3,4}$ 


\section{Introduction}

Laparoscopic surgery has become increasingly mainstream in recent times on account of the reduced discomfort and the reduced recovery time following surgery. However, the use of such surgical procedures represent significant challenges to the surgeons on account of a wide range of restrictions compared to traditional open surgery. ${ }^{5}$

The benefits of laparoscopic surgery stem from the reduced net length of incision, typically multiple small incisions are made to gain access to the surgical site. Typically, a 12 $\mathrm{mm}$ port ( $\sim 15 \mathrm{~mm}$ Trocar $)$ is provided for a rod-lens camera, and two $5 \mathrm{~mm}(\sim 9 \mathrm{~mm}$ Trocar) ports are used for surgical forceps and a total incision length of about $33 \mathrm{~mm}$. Furthermore, multiple smaller incisions result in less discomfort and faster recovery compared to single extended incisions of the same net length.

The challenges presented by laparoscopic surgery originate from the limited access, limited viewability, limited dexterity, significant loss of haptic feedback and the increased complexity or nonintuitive operation of many of the laparoscopic surgical instruments that are currently available. This situation demands that surgeons must have increasingly high levels of training to be able to make use of laparoscopic surgery in a safe and efficient manner. ${ }^{6}$

As a result of the above considerations, most operations of a simple nature will be initiated and completed using laparoscopic surgery, however, in the case that unforeseen complications are encountered open surgery may be resorted to, to make it easier for the surgeon to work, as well as provide increased safety in regard to the inherently increased site access, viewability and haptic feedback. Therefore, surgeons must be proficient in the use of regular surgical instruments as well as an increasing range of laparoscopic instruments. ${ }^{7}$

In order to increase the operating efficiency as well as increase the capabilities of laparoscopic surgery, it is essential to develop laparoscopic instruments that are inherently easier to use, more intuitive to use and have increasing capabilities despite the limitations. An essential part of this process is for surgeons and engineers to closely work together. In regard to the development of such instruments on one hand, it is important for the medical staffs to share difficulties that they encounter, and on the other hand, it is equally important for engineering staff to share new technologies that have been developed that could potentially contribute to the development in medical engineering. ${ }^{8}$

This close collaboration between medical and engineering staff is essential to the improvement of instruments such as surgical instruments. Typically, a specific need will be expressed by medical staff and together they will discuss possible solutions. In the case of a surgical instrument, this may be designed in three-dimensional (3D) CAD and a concept prototype 3D printed. This allows for direct feedback from the medical staff in the shortest possible period of time. This process facilitates iterations until instrument feasibility is confirmed, at this point, manufacture of a fully functional instrument will be made, and can be immediately tested in experiments such as a porcine experiment. In the case of laparoscopic instruments, while a conceptual model can easily be printed providing medical staff with a general feel for the size and shape of the instrument, the inherent microscale and strength of materials far exceed the capabilities of current $3 \mathrm{D}$ printers. ${ }^{9}$ 


\section{Methods}

\subsection{Laparoscopic retractors --- ideals}

The retractor as mentioned above is one of the many essential laparoscopic instruments. It provides a device to temporarily hold such as an organ or vessel to allow access to an area that is obstructed in some way by the organ or vessel. In the case of open surgery, the above-mentioned retraction can be achieved directly by hand or using any convenient instrument. The ideals of retraction are meant for the device to be suitably sized to match the size and shape of the target requiring retraction. The device must also provide a suitable pressure and friction during retraction for the specific task.

In the case of laparoscopic retraction, the ideal shape and size is difficult to achieve as the mechanism most ideally should be able to pass through a $5 \mathrm{~mm}$ or $10 \mathrm{~mm}$ trocar. In regard to suitable pressure, the limited viewing typically provided by a rod lens and lack of direct haptic feedback make the judgement of an appropriate use of pressure difficult. ${ }^{10,11}$

\subsection{Current retraction practices}

At the time of writing, local surgeons indicate that they use the regular forceps "as is" for retraction and if the retraction is insufficient, they indicated that they take the forceps out, grasp a gauze and wrap it around neatly to allow reinsertion through the trocar. This typically works well until the gauze becomes soaked with blood or body fluids and requires replacement. ${ }^{9}$

Another common alternative is a very small, low cost, simple and disposable swab (Ethicon $5 \mathrm{~mm}$ and $10 \mathrm{~mm}$ Dissectors) commonly referred to as a "cherry dissector", shown in Fig.1. Both $5 \mathrm{~mm}$ and $10 \mathrm{~mm}$ versions are shown in Figs. 1(a) and 1(b), respectively. This is disposable and is similar to a large cotton bud in texture and functionality - it must be noted, however that such dissectors are targeted as swabs. (A similar product, the Endo Peanut ${ }^{\mathrm{TM}}$ by Covidien instrument is

Fig. 1. (a) Endostik ${ }^{\circledR}$ Bullet $5 \mathrm{~mm}$ and (b) Endostik ${ }^{\circledR}$ Cherry $10 \mathrm{~mm}$.

Source: Image Fabco.net.

designed for endoscopic surgery for swabbing small amounts of fluid or for blunt dissection of soft tissue(s) and structures, or as an aid in controlling minor intraoperative bleeding. $)^{12}$

Such cherry dissectors although not designed retractors are effective for minor retraction needs.

Clearly for a retractor tip to exceed $10 \mathrm{~mm}$ in diameter, some kind of articulated mechanism or balloon is required. ${ }^{13}$

Some examples of commercially available "expandable" retractors are the "Endo paddle" by Covidien and the "Cat hand" by Hakko.

Figure 2 shows an Endo paddle (Covidien). The tip passes through a $10 \mathrm{~mm}$ port and expands as shown in Fig. 2 to a width of up to $80 \mathrm{~mm}$, a covering fabric provides a net and 
traction for the organ being handled. The frame of the Endo paddle is metallic and supports significant weight/pressure.

Figure 3 shows a $5 \mathrm{~mm}$ and a $10 \mathrm{~mm}$ version of the Cat hand (Hakko) which expand to $30 \mathrm{~mm}$ and $38 \mathrm{~mm}$, respectively. The Cat hand uses a balloon-like mechanism that is pumped up with air to full size upon insertion through the trocar. The lack of any frame limits the effective weight/pressure that can be applied to the Cat hand retractor.

Thoracic surgeons at the Nagasaki University hospital requested the Medical Engineering Hybrid program staff to develop a retractor suitable for lungs. As a

Fig. 2. Endo paddle.

Fig. 3. Cat hand.

result, a number of models have been designed, prototyped and tested and are outlined in the following section.

\subsection{Developed models}

Regarding target specifications of the retractor, the mechanism must be capable of stably holding the lung or lung segments as required. Ideally, a $5 \mathrm{~mm}$ port should be targeted.

Firstly, stable retraction of the lungs is considered. Lungs are inherently delicate, soft and slippery to handle on account of body fluids. Therefore, ideally, the retractor should provide a shape that is well suited to the safe handling of lungs as well as provide a surface that provides optimal traction.

Initially, a larger version of the Cherry dissector was considered. Therefore, an inflatable balloon was created as shown in Fig.4. This development was carried out in cooperation with the Ashimori Industry Co. Ltd. Since this initial prototype, a number of improved models have been created.

The first prototype model is shown in Fig. 4 which consisted of a rubber balloon attached to a stainless steel pipe suitable for use with a $10 \mathrm{~mm}$ trocar. By inflating the balloon, the appropriate size for retraction could be obtained after insertion.

Figure 5 shows the first balloon retractor used for lung retraction during a porcine experiment. While the expanded diameter of approximately $4 \mathrm{~cm}$ provided the easy manipulation of the lung, the particular rubber material used proved to be too slippery for practical application. The type of rubber used was butyl rubber.

Figure 6 shows the second balloon retractor model. Figure 6(a) shows the deflated state for insertion through a trocar and Fig.6(b) shows the fully inflated state for retraction. Improvement focused on the use of a slip resistant-type of silicon rubber and the reduction of overall diameter to about $6 \mathrm{~mm}$, which would be

Fig. 4. Balloon retractor first model.

Fig. 5. Porcine experiment of the first balloon retractor model.

Fig. 6. (a) Balloon retractor second model — deflated and (b) second model tip close up image — inflated. 
suitable for a $5 \mathrm{~mm}$ (actual internal diameter of about $6 \mathrm{~mm}$ ) port. Inflation was provided for using a sphygmomanometer bulb-type pump and valve.

Figure 7 shows the second balloon retractor in use for retracting a lung during a porcine experiment. Though the tip of this model is smaller than the first model - about $2.3 \mathrm{~cm}$, it's retraction was sufficient. The non-slip silicon proved to result in notably less slip compared to the first model. However, the protruding section of about $14 \mathrm{~mm}$ as seen in Fig. $6(\mathrm{~b})$ proved to be a hindrance to lung retraction.

Fig. 7. Porcine experiment of the second balloon retractor model.

Fig. 8. Third balloon retractor model — partially inflated.

Figure 8 shows the third retractor model. Improvement focused on reducing the protruding section as much as possible to $5 \mathrm{~mm}$, and the addition of an elastic tubular net cover made of nylon 6,6 to increase the traction. This addition of the net resulted in providing additional retraction, but the protruding section although shorter continued to be a hindrance.

Figure 9 shows the third balloon retractor in use for retracting a lung during a porcine experiment - partial inflation was sufficient for porcine lung retraction.

Figure 10 shows the forth and most recent model at the time of writing. This time, the improvement focused on the elimination of the protrusion at the tip and an additional overall retracting length was requested. Porcine experimentation has yet to be performed at the time of writing.

Regarding the development of the retractor, to this point, only subjective analysis by surgeons during porcine experiments has been obtained. Therefore, in the following section, objective analysis of the respective retractors have been

Fig. 9. Porcine experiment of the third model.

Fig. 10. Forth balloon retractor model.

carried out to gauge absolute static traction on a model lung. Clearly, both subjective and objective data are necessary in evaluation of the respective retracting mechanisms.

\subsection{Measurement of static frictional coefficient}

In order to provide some degree of objectivity in evaluating the respective retractors, the static frictional coefficient of each retractor was measured for both wet and dry conditions. In the case of retracting a lung, only the static parameters were considered, however dynamic measurement of the frictional coefficient is outlined in Isami et al. ${ }^{14}$

An image of the measurement procedure is shown in Fig. 11. Figure 12 shows a photo of the actual equipment used for measurement, and Fig. 13 shows a close up of the loading detail.

Regarding how much pressure was considered appropriate for each receptor, a two-step approach was used. Firstly, pressure was chosen by what felt an appropriate maximum loading for each device and this loading was noted. Next, based on an understanding of the 
relationship between loading and area, an average per square centimeter was decided upon and all measurements were normalized based on this.

Using this average pressure per square centimeter loading of $5 \mathrm{~g} / \mathrm{cm}^{2}\left(\sim 0.049 \mathrm{~N} / \mathrm{cm}^{2}\right)$ was used for testing each of the retractors for both dry and wet measurements.

Fig. 11. Image of measurement procedure.

Fig. 12. Overall photograph of measurement procedure.

Fig. 13. Enlarged view of loading shown in fig. 12.

Furthermore, $+/-50 \%$ measurements were also taken to confirm the reliability of the readings, thus each receptor was measured at $2.5,5$ and $7.5 \mathrm{~g} / \mathrm{cm}^{2}$ per reading. The value of 5 $\mathrm{g} / \mathrm{cm}^{2}$ perhaps sounds very low, but was judged appropriately as the pressure was required to stably hold a lung without excessive pressure.

The procedure used was as follows: each retractor is balanced on the trolley (set to an equilibrium) so as to exert no pressure at the tip and taped into position as can be seen in Fig. 13. The resistance of the trolley wheels was checked to be negligible compared to that of the resistance under measurement.

Regarding the calculation of the static frictional coefficient, the above-mentioned loading was normalized to $5+/-50 \% \mathrm{~g} / \mathrm{cm}^{2}$ and is the contact pressure $P$ and the area that makes contact with the lung is $A \mathrm{~cm}^{2}$. The normal direction force $N$ was calculated by multiplying the contact pressure $P$ and the contact area $A$ between the tip of the retractor and the lung model as follows $N=P A$, where $N$ is the normal direction force $(\mathrm{g}), P$ : the pressure of the contact area $\left(\mathrm{g} / \mathrm{cm}^{2}\right)$ and $A$ is the contact area $\left(\mathrm{cm}^{2}\right)$.

The retractor was connected to the spring gauge through the pulley as shown in Figs. 11 and 12. The maximum static frictional force $F$ is the scale reading of the spring gauge at the moment that the tip of the retractor slips on the lung model after the tip of the retractor is dragged across the lung model.

The static frictional coefficient is calculated by $\mu=F / N$, where $\mu$ is the static frictional coefficient (-), $F$ is the maximum static frictional force $(\mathrm{g})$ and $N$ is the normal direction force $(\mathrm{g})$.

\section{Result and discussion}

The results of the measured static frictional coefficients for the case of $\mathrm{P}=5 \mathrm{~g} / \mathrm{cm}^{2}$ are shown in Table1. Three retractors are those developed by the authors and the following five commercially available retractors were used for comparison. The second model developed was overinflated and burst and was not available for frictional testing.

The "wet" experiments were carried out with saline solution to emulate the natural body fluids that are present during laparoscopic surgery.

With the exception of the Cherry dissectors which provide a very limited retracting area, the developed models provided excellent retraction compared to

Table 1. The comparison of static frictional coefficients for $P=5 \mathrm{~g} / \mathrm{cm}^{2}$. 
currently available retractors in regard to traction as indicated by the measured static frictional coefficients. Therefore, the newly developed models are expected to provide increased retracting efficiency.

At the time of writing, Cherry dissectors are commonly used for retraction for endoscopic, laparoscopic and thoracoscopic surgery. The factors that contribute most to this trend are perhaps the simplicity of use and relatively high traction provided.

Comparing each of the commercially available products, the Cherry dissector had the highest static frictional coefficient (1.8-2.4 wet), followed by the Cat hand (0.9-1.1 wet) and finally the Endo paddle ( 0.7 wet) (refer to Table 1 and Fig. 14). Table 1 also indicates the actual total pressure exerted on the device to provide an indication of the scale of the pressure ranging from $\sim 0.06 \mathrm{Nm}$ for the Cherry dissector to $\sim 3.36 \mathrm{Nm}$ for the Endo paddle.

Regarding the developed models (Table 1 and Fig. 14), the rubber balloon retractor exhibited the highest static frictional coefficient, followed by the 3rd model and finally the latest model. The reason for the significant drop between the 3rd and 4th models may be due to the shape, that is a shorter balloon sinking in to the lung to some degree as was also observed in the case of the $10 \mathrm{~mm}$ Cherry dissector and thus on account of the shape providing additional traction. Furthermore, upon careful observation of the nylon 6,6 net used on both the models, a slight variation in the fine net pattern was observed and this aspect requires further investigation.

Fig. 14. Graph of static frictional coefficients dry versus wet by retractor.

In regard to comparison between dry and wet readings, for some retractors, the static frictional coefficient increased while others decreased, as clearly observed in the graph in Fig. 14. This is most likely on account of a combination of the tractive qualities of the surface material and the shape of the retractor.

Regarding the results of the $5 \mathrm{~mm}$ Cherry dissector, although the raw data show the best result, as mentioned in the notes of Table1, the small area and resulting low pressure could not be measured reliably on the $-50 \%$ reading and the high wet reading may be influenced by capillary pressure. The $10 \mathrm{~mm}$ Cherry dissector provided the best retraction amongst the currently, commercially available products and this is most likely a combination of both the optimized shapes - spherical and surface texture (cotton - Fig. 1(b)). However, the size is insufficient to effectively retract a lung segment, thus based on this and the data overall, perhaps the developed model 3 is optimal in terms of size $\sim 1.5 \mathrm{~cm} \mathrm{X} 2.5 \mathrm{~cm}$ and surface cover (nylon 6,6 net) for retraction of a lung or lung segment, providing that the protruding tip can be eliminated. It is therefore expected that a modified version of model 3 would be optimum for retraction of a lung or lung segments.

Other aspects that must be considered in the case of retractors are obstruction of the camera, that is consideration for viewability, in the case of a balloon, clearly some obstruction will be inherent compared to lower profile retractors as those currently available, furthermore, in the case of a balloon, safety and potential bursting of the balloon must be considered, particularly when being used in close proximity to sharp- or heat-based cutting instruments. In the case of using a net covering, any potentially separated fragments would be contained. 


\section{Conclusion}

A balloon-based organ retractor is in the process of being developed for laparoscopic surgery. Initially, the retractor was trialed during porcine experiments and positive feedback was provided by surgical staff. However, in order to provide a quantitative evaluation of the new instruments, the static frictional coefficient was measured on a model lung and the data were compared with the currently used commercially available retractors.

With the exception of Cherry dissectors which provide a limited area of retraction, the retractors under development exhibited improved retraction. Through the prototyping of a variety of models, optimum shape, size and texture have been better understood.

While the most recently developed model has yet to be trialed in a porcine experiment, it is expected to provide for the improved retraction compared to the currently available retractors. The improved efficiency in retraction will lead to improved operating efficiency and safety for laparoscopic operating procedures.

\section{Acknowledgment}

The authors wish to express their appreciation to Mr. Hideki Yamashita of Ashimori Industry Inc. for his cooperation in making the prototype models.

\section{References}

1. Okamoto K, Yozu R, Designing innovative retractors and devices to facilitate mitral valve repair surgery, Ann Cardiothorac Surg 4(4):364-369, 2015.

2. Hashimoto D, Current problems and its solutions in minimally invasive surgery, Modern Media 50(12):7, 2004.

3. Palanivelu P, Patil KP, Parthasarathi R, Viswambharan JK, Senthilnathan P, Palanivelu C, Review of various liver retraction techniques in single incision laparoscopic surgery for the exposure of hiatus, J Min Access Surg 11(3):198-202, 2015.

4. Hamed O, Gusani NJ, Kimchi ET, Kavic SM, Minimally invasive surgery in gastrointestinal cancer: Benefits, challenges, and solutions for underutilization, J Soc Laparoendosc Surg 18(4):e2014.00134, 2014, doi: 10.4293/JSLS.2014.00134.

5. Rao PP, Rao PP, Bhagwat S, Single-incision laparoscopic surgery - current status and controversies, J Min Access Surg 7(1):6-16, 2011.

6. Litynski GS, Mouret, Dubois, and Perissat: The laparoscopic breakthrough in Europe (19871988), J Soc Laparoendosc Surg 3(2):163-167, 1999.

7. Perugini RA, Callery MP, Complications of laparoscopic surgery, in Holzheimer RG, Mannick JA (eds.), Surgical Treatment: Evidence-based and Problem-oriented, Zuckschwerdt, Munich, 2001.

8. Beira RD, Dexterous mechanical systems for intuitive telemanipulation in minimally invasive surgery, Ph.D. Thesis, Institute for Systems and Robotics, Lisboa, Portugal, 2013.

9. Yamamoto I, Ota R, Zhu R, Kondo M, Lawn M, Nagayasu T, Yamazaki N, Matsumoto K, Research and development of surgical instruments based on biomechanical technology, Proc SMIT, Design of Medical Devices, Tracking \& Smart Tools, pp. 32, 2016.

10. Westebring-van der Putten EP, Goossens RHM, Jakimowicz JJ, Dankelman J, Haptics in minimally invasive surgery — A review, Minim Invasive Ther Allied Technol 17:3-16, 2008. 
11. Toshio K, Takeshi Y, Mitsutoshi N, Tetsuyou W, Incompressible liquid based force sensible silicone retractor attachable to surgical suction instruments, Proc IEEE/ ASME Int Conf Advanced Intelligent Mechatronics (AIM), pp. 1398-1404, 2016.

12. Available at: http://products.covidien.com/pages. aspx?page=ProductDetail\&id= 13468\&cat=Devices\&cat2=Model, Accessed on 1 May 2017.

13. Yamamoto I, Kishikawa K, Lawn M, Nagayasu T, Yamazaki N, Matsumoto K, Development of the surgical instruments using multi-joint mechanism in endoscopic surgery, Proc IMETI 2016, pp. 1-6, 2016.

14. Isami N, Yosuke T, Satoshi N, Noboru T, Frictional characteristics of clamp surfaces of aneurysm clips finished by laser processing, J Adv Mech Des Syst Manuf 10(2): JAMDSM0026JAMDSM0026, 2016. 
Table 1

\begin{tabular}{|c|c|c|c|c|}
\hline \multirow{2}{*}{ Retractor type } & \multicolumn{2}{|c|}{$\begin{array}{l}\text { Static frictional } \\
\text { coefficient }\end{array}$} & \multirow{2}{*}{$\begin{array}{c}\begin{array}{c}\text { Approximation } \\
\text { net } \\
\text { Pressure- }\end{array} \\
\text { to lung }\end{array}$} & \multirow{2}{*}{$\begin{array}{l}\text { Retracting area } \\
\text { (s=shaft-area) } \\
\text { (width x length) }\end{array}$} \\
\hline & $\begin{array}{c}\text { Dry- } \\
\text { lung- } \\
\text { model }\end{array}$ & $\begin{array}{l}\text { Wet- } \\
\text { lung- } \\
\text { model }\end{array}$ & & \\
\hline $\begin{array}{l}\text { Developed-Rubber- bal- } \\
\text { loon--1st model }\end{array}$ & 2.91 & 2.27 & $\sim 0.4 \mathrm{~N}+/-50 \%$ & $\sim 8 \mathrm{~cm}^{2} \cdot(2.5 \mathrm{x} \cdot 3 \mathrm{~cm})-$ \\
\hline $\begin{array}{l}\text { Developed-Silicon- bal- } \\
\text { loon-with - elastic tubular- } \\
\text { net-3rd model }\end{array}$ & 1.66 & 1.98 & $\sim 0.19 \mathrm{~N}+/-50 \%$ & $\sim 6 \mathrm{~cm}^{2} \cdot(1.5 \times 2.5 \mathrm{~cm})$ \\
\hline $\begin{array}{l}\text { Developed Elongated- } \\
\text { silicon- balloon with- } \\
\text { elastic tubular- net- } 4 \text { th- } \\
\text { model }\end{array}$ & 0.98 & 0.98 & $\sim 0.69 \mathrm{~N}+/-50 \%$ & $\sim 14-\mathrm{cm}^{2} \cdot(2 \times 6 \cdot \mathrm{cm})$ \\
\hline Cherry dissector $5 \mathrm{~mm}$ & $0.67^{* 3}+4$ & $2.39^{\circ 4}$ & $\sim 0.06 \mathrm{~N}+/-50 \%$ & $\sim 1^{* 1} \mathrm{~cm}^{2}(0.5 \times 1 \mathrm{~cm}+\mathrm{s})$ \\
\hline Cherry dissector $10 \mathrm{~mm}$ & 2.32 & 1.77 & $\sim 0.07 \mathrm{~N}+/-50 \%$ & $\sim 1.5 \mathrm{~cm}^{2} \cdot(1 \mathrm{~cm}$ sphere $)$ \\
\hline Endo paddle $10 \mathrm{~mm}$ & 0.56 & 0.73 & $\sim 3.36 \mathrm{~N}+/-50 \%$ & $\sim 67^{* 2} \mathrm{~cm}^{2}(7.5 \times 8.9 \mathrm{~cm})$ \\
\hline Cat hand $5 \mathrm{~mm}$ & 1.13 & 1.07 & $\sim 0.33 \mathrm{~N}+/-50 \%$ & $\sim 6 \mathrm{~cm}^{2} \cdot(2.5 \times 2.5 \mathrm{~cm}+\mathrm{s})$ \\
\hline Cat hand $10 \mathrm{~mm}$ & 0.74 & 0.90 & $\sim 0.39 \mathrm{~N}+/-50 \%$ & $\sim 12 \mathrm{~cm}^{2} \cdot(3.5 \mathrm{x} \cdot 3.5 \mathrm{~cm}+\mathrm{s})$ \\
\hline \multicolumn{5}{|c|}{ 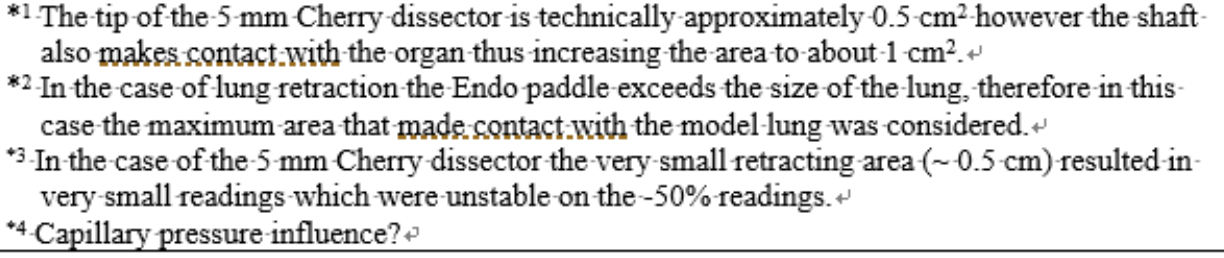 } \\
\hline
\end{tabular}


Figure 1a

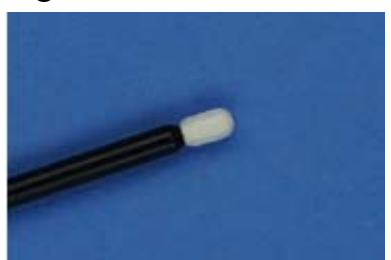

Figure $1 \mathrm{~b}$

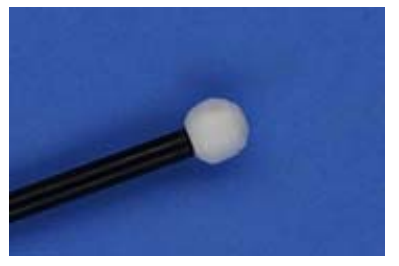

Figure 2

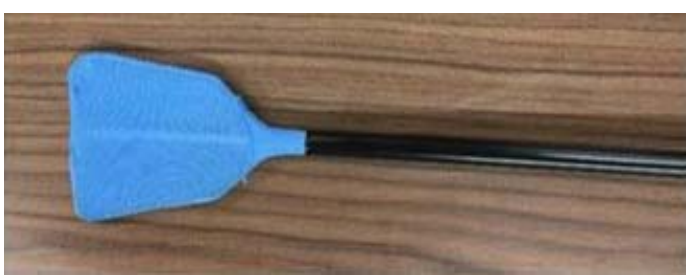

Figure 3

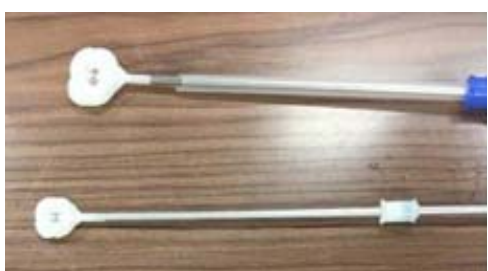

Figure 4 
Figure 5

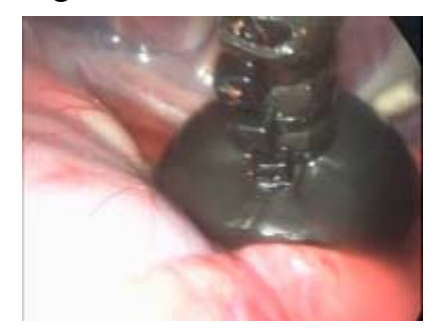

Figure 6a

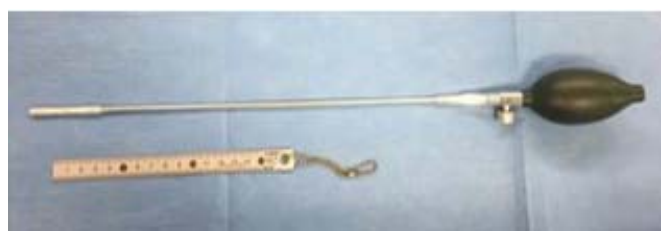

Figure 6b

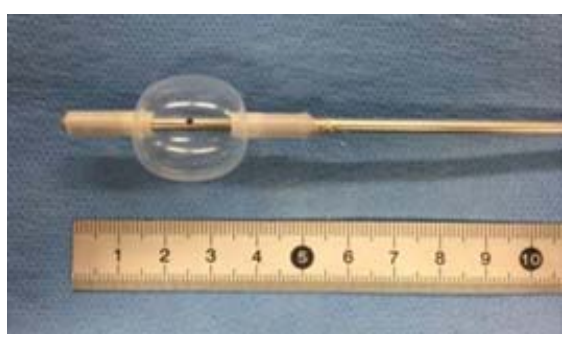

Figure 7

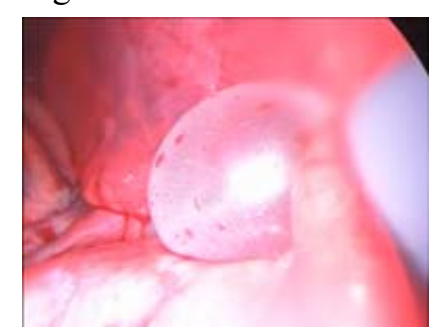


Figure 8

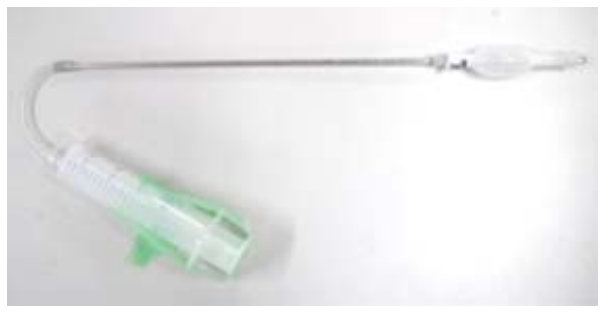

Figure 9

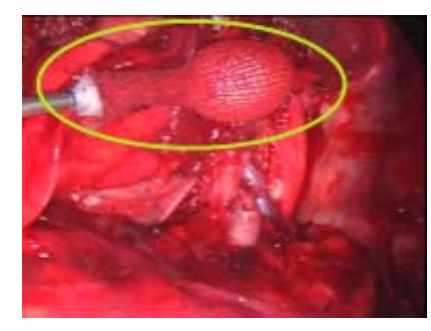

Figure 10

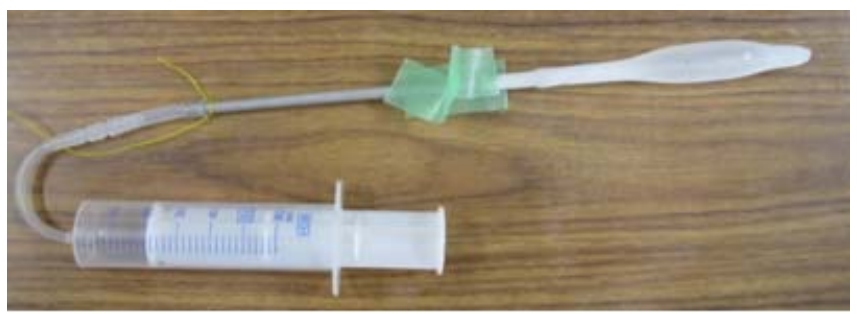

Figure 11

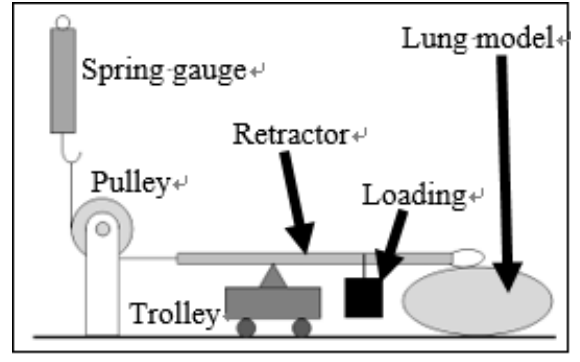


Figure 12



Figure 13

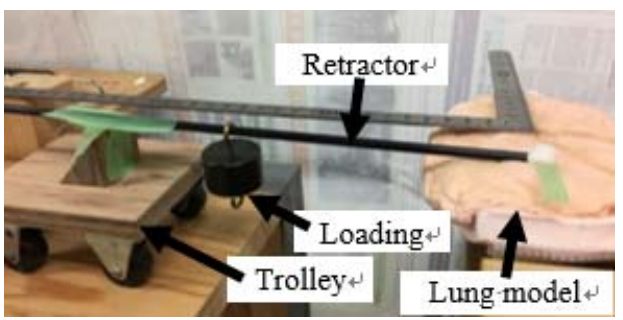

Figure 14

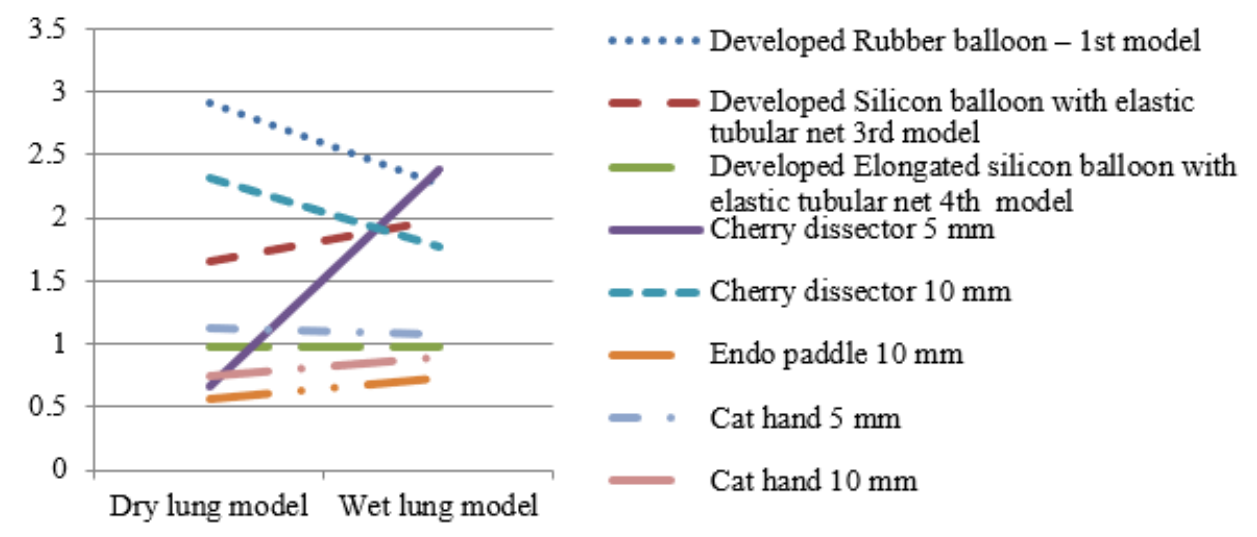

